\title{
iMatrixSpray: A Free and Open Source Sample Preparation Device for Mass Spectrometric Imaging
}

\author{
Markus Stoeckli*, Dieter Staab, Michael Wetzel, and Matthias Brechbuehl
}

\begin{abstract}
A device was built for matrix deposition in mass spectrometric imaging. This spray-type instrument requires no user interaction other than providing the spray solution and selecting the pre-defined or custombuilt method. Robustness was achieved by utilizing a delta-robotics design in combination with a simple liquid system. All the information describing the systems is provided as open source and hardware and the design is therefore suitable for wide distribution and adaption by the scientific community.
\end{abstract}

Keywords: MALDI - MALDI MSI - Mass spectrometric imaging · Matrix deposition · Sample preparation

\section{Introduction}

With the extension of matrix-assisted laser desorption/ionization mass spectrometry (MALDI MS) into imaging mode, ${ }^{[1]}$ a new research field opened up with immense opportunities to provide label-free molecular imaging capability previously not accessible. Instead of acquiring one mass spectrum from a liquid sample, the same mass spectrometers are used in rastering mode, acquiring thousands of spectra from an array of positions, allowing the depiction of the spatial distribution of the acquired analytes. This extension required the modification of existing acquisition protocols, including the control of the sample stage, modification of data acquisition software and programming of data processing and visualization routines. While these steps were at the beginning perceived as major hurdles and later recognized as enabling achievements, it is in our view sample preparation that is the key to the success of MS imaging.

\footnotetext{
${ }^{*}$ Correspondence: Dr. M. Stoeckli

Novartis Institutes for Biomedical Research

WSJ-155.2.27, Novartis Campus

$\mathrm{CH}-4056$ Basel

Tel.: +4161324 7743

E-mail: markus.stoeckli@novartis.com
}

Mass spectrometric imaging (MSI) ${ }^{[2]}$ depicts the spatial distribution of molecules on a surface, and it does this by requiring no labeling of the molecules and little sample preparation. Secondary ion mass spectrometric (SIMS) or laser desorption/ionization (LDI) imaging are the most direct way of MSI, as the sample can be introduced and analyzed in the mass spectrometer without addition of matrix or chemicals, but they are lacking sensitivity for high-mass biomolecules, limiting its application in biomedical research. In contrast, MALDI MSI gained popularity due to its unmatched sensitivity for biomolecules, permitting analysis of many substance classes including lipids and proteins directly from biological tissue. But, it has the intrinsic disadvantage of requiring a matrix to be applied to the sample before analysis. In the traditional MALDI experiment, the dissolved analyte is mixed with a matrix solution and allowed to slowly co-crystallize. While this process is suitable for single samples, it is not directly transferable to an imaging experiment, where the spatial distribution of the molecules in the original samples relative to each other is to be preserved. In MS imaging, the matrix application is also the extraction step for the molecules out of the substrate, contributing most significant to the success of an imaging experiment. In this respect, the requirement of a perfect matrix application protocol is to efficiently extract the analytes of interest out of the sample while preserving their relative position in the imaging plane, and to slowly co-crystalize the analyte with the matrix to result in highest sensitivity in the MALDI MS measurement. While the description of the task at hand is very simple, it has been a challenge since the beginning of MALDI MS imaging and many man-years have been spent on the development of adequate methods and devices to allow a reproducible sample preparation which is also suitable for automation.

This article describes the design and operation of a fully automated matrix deposition device, which is distributed under the free and open source hardware concept. It is based on widely available components and was developed for the specific requirements of matrix deposition. It is a fully automated system, facilitating push-button coating of samples with no user intervention. The device was tested in a laboratory environment and proved to reliably contribute to high-quality MS imaging data.

\section{Background}

The range of the published matrix deposition protocols is wide, starting with simple drop deposition by a manual pipette to application of commercial devices. This article does not cover all the attempts to develop the best method, as there are reviews available on sample preparation for MALDI MS imaging also covering matrix deposition. ${ }^{[3-5]}$ To illustrate the complexity of the task at hand, these are a few selected examples of what has been tested so far: The use of the water molecules already present in the biological tissues as matrix for infrared lasers has been tried since the beginning of MS imaging and new results were published recently. ${ }^{[6]}$ Since the solvent in the matrix solution leads also to horizontal distribution of the analytes, solvent-free matrix application protocols have been developed and applied to study lipid distributions. ${ }^{[7-9]}$ For thin samples, modified surfaces with a matrix effect (e.g. silver nanoparticles) were tested. ${ }^{[10,11]}$ There is a variety of spray solutions to be found in 
literature, ranging from manual spray, oscillating capillary ${ }^{[12]}$ to automated spray. ${ }^{[13]}$ These were combined with conductive surfaces ${ }^{[14]}$ or gold coating ${ }^{[15,16]}$ for use in in time-of-flight instruments. Multiple devices have been reported to deliver small drops in an array, using inkjet technology[17] or acoustic wave drop generation. ${ }^{[18]}$ These and many other approaches are currently applied to sample preparation for MALDI MSI, where they facilitate measurement of specific compound classes and sample types. Despite the availability of these methods and devices, the search continues for a system with improved performance for this task.

\section{Design Concept}

The aim of the work published here was to build a robust and open system, based on past experience and open for new development. For this reason, a device was constructed and is released to the scientific community under the free and open source hardware concept. ${ }^{[19]}$ This open concept directed the development of the apparatus, as it was built using commonly available and relatively inexpensive components. Founded on previous success with manual and pneumatic spray in our laboratory and published work, ${ }^{[13]}$ the fundamental concept of spray deposition was selected.

Besides the obvious requirements of being able to reproducibly and homogeneously deliver a matrix layer, the design is to avoid contamination of mechanical parts and facilitate automatic cleaning without manual intervention, as well as selection of multiple deposition reagents for it to be used for internal standards or on-surface peptide digest, just to list a few potential applications. Fig. 1 shows the building blocks of the device, described in detail below. The mechanical drawings as well as the software can be downloaded from the dedicated website. ${ }^{[20]}$

\section{Mechanics}

The core of the system is a delta robotics stage, a concept which is described in detail in the thesis of Clavel.[21] This design has the benefit of merely requiring 3 motors and 10 mechanical parts to build a complete xyz robotic system. The design limits contamination, as the motors are well separated from the spray. The final dimensions of the arms were selected to allow a movement of 20 by $20 \mathrm{~cm}$ in the spraying pane, with a spatial resolution of better than $0.1 \mathrm{~mm}$. This was found to be the optimal geometry for coating a single standard SBS-sized plate without restricting travel in any dimension. The upper arms with a

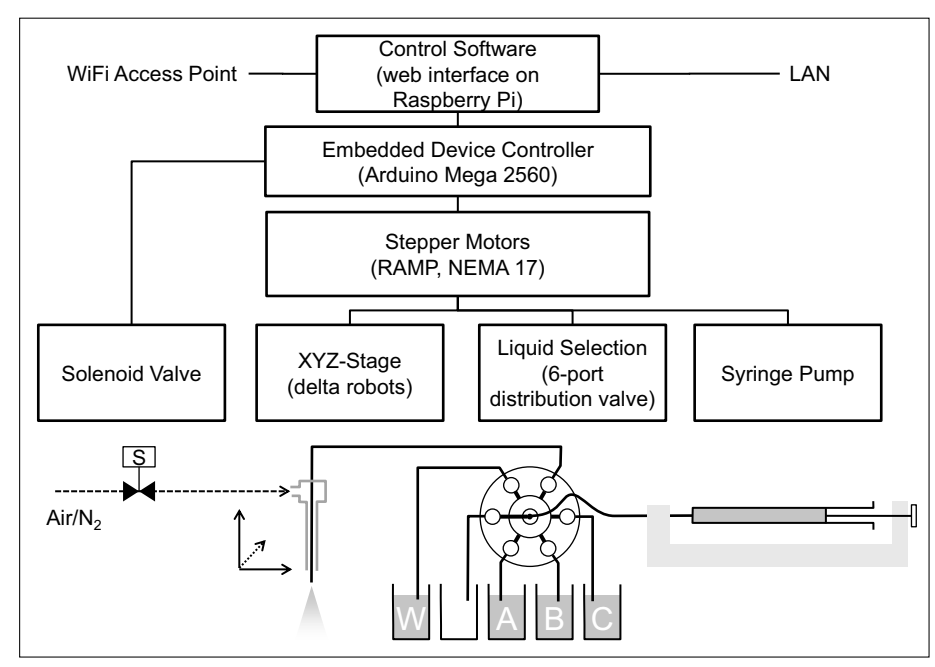

Fig. 1. iMatrixSpray design concept. The device operates as WiFi access point and the user controls the system though a web interface. Opensource components build the hardware and software layers of the device, allowing the deposition of up to three solutions ( $A$ to $\mathrm{C}$ ) and automatic rinsing with a wash solution (W). length of $71.25 \mathrm{~mm}$ are directly attached to generic NEMA 17 stepper motors. From there, $3 \times 2$ parallel rods of $170 \mathrm{~mm}$ length connect the moving platform with clip-on ball joints. All the electronics rest above the motors and the mechanical parts can be removed for cleaning without any tools.

\section{Spray and Liquid System}

Attached to the moving platform of the delta robotics actuator is a pneumatic spray nozzle, built by a fused silica capillary with an inner diameter of $180 \mu \mathrm{m}$ (Infochroma, Zug, Switzerland) and a coaxial stainless tube of $0.75 \mathrm{~mm}$ inner diameter (VICI, Schenkon, Switzerland). The end of the capillary can be adjusted to produce a stable and homogenous spray cone, depending on the liquid flow and the gas pressure. Good results were achieved by reaching the capillary end $0.5 \mathrm{~mm}$ out of the outer tube, in combination with a gas pressure of 1.2 bar.

The liquid flow was generated by a syringe pump, holding a syringe of $1 \mathrm{~mL}$ volume (Hamilton, Bonaduz, Switzerland) and a motorized 6-port medium pressure selection valve (V-241, Upchurch, Oak Harbor, WA). The syringe is connected to the central port (see Fig. 2), whereas the capillary and the three solution containers as well as wash solution container and a waste container were connected to the outer ports. This flexible setup allows fully automatic operation and rinsing.

The nearly saturated matrix solution poses a significant issue for matrix deposition as it has the potential to crystallize in the system leading to clogging or unevenspray. The device prevents this by rinsing the capillary and nozzle with methanol after each spray deposition. The nozzle cleaning procedure involves slow delivery of methanol without engaging the coaxial air flow. The surface tension encourages the methanol to build drops at the end of the spray, which soak up to the outer capillary, thereby dissolving any remaining matrix. The drops fall in a waste reservoir and the nozzle is dried by engaging the coaxial air flow at the end of the cleaning procedure.

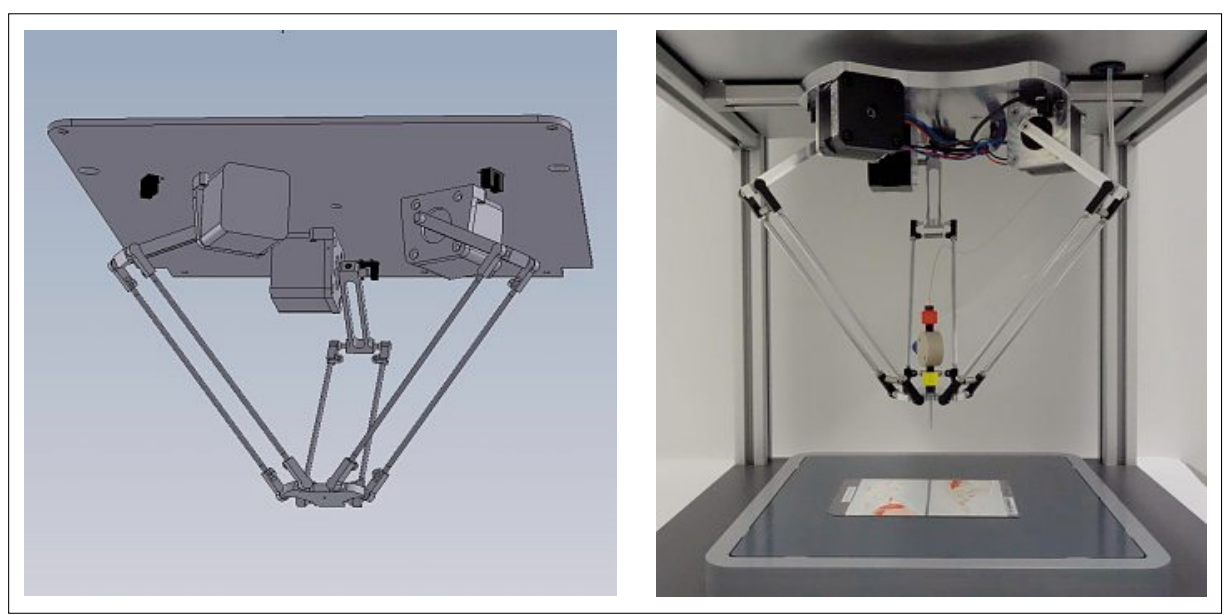

Fig. 2. Delta robotics configuration using three stepper motors to control the three axis of translation. Design (left) and actual implementation (right), able to coat an area of $20 \times 20 \mathrm{~cm}$. 


\section{Electronics and Software}

The popular Arduino platform ${ }^{[22]}$ forms the core of the matrix deposition system. Attached to the Arduino Mega 2560 is a RAMP module ${ }^{[23]}$ which connects to the stepper motors with the end stops and the solenoid valve. The web interface was realized on a Raspberry $\mathrm{Pi}^{[24]}$ Linux system with a Wi-Fi dongle and a camera module. The software is based on open source code of Marlin ${ }^{[25]}$ and OctoPrint, ${ }^{[26]}$ with the actual software being available for download at iMatrixSpray.com. Power is provide to the device by an external 12 V/8 A power supply. By using these components, the cost of the electronics to build the system is below $500 \mathrm{CHF}$.

\section{Operation}

The current implementation of the software provides two fundamental modes of operation. First, and most simple, is the parametric mode where the spray is controlled by entering a few values for the spray definition (Fig. 3). For a typical experiment, the user connects to the device through Wi-Fi and is presented with the web interface. If new liquids are added, the system is primed using a single click. The spray solution is selected as well as the desired matrix density and the number of coating cycles. Upon clicking on the Spray button, the coating process is executed and the spray rinsed afterwards without further user interaction.

As a second mode, the software provides the ability to directly upload and execute gCode files to operate the device using basic commands. This provides flexibility to define more complex experiments, including for example tryptic digest with enzyme, buffer and matrix deposition. Spray cycles with different lengths, deposition of standard drops or drying using the nozzle are just a few examples which are possible.

\section{Performance}

The optimization of matrix deposition is a multi-parametric problem, with the difficulty that the only meaningful readout is the quality of the final MS image. Optical inspection of the spray surface may only deliver readout on the homogeneity and the reproducibility of the matrix layer only. The selected components (computer-controlled xyz stage and syringe pump) guarantee that these parameters are constant and experiments with matrix layers on transparent films confirm this assessment (data not shown).

Based on the experience from manually spraying matrix, a deposition protocol

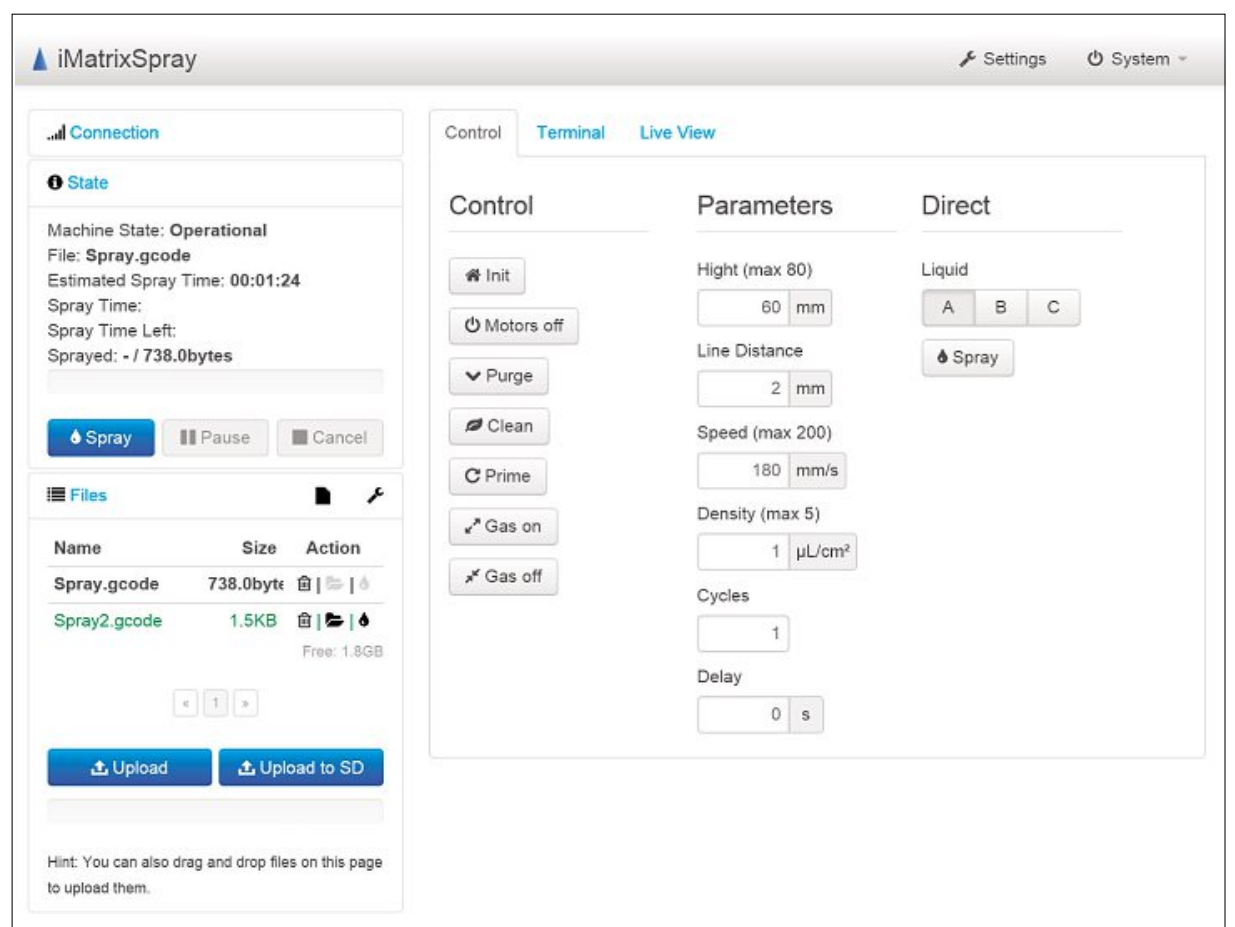

Fig. 3. iMatrixSpray user interface. Parametric and scripting modes are available.

was developed and optimized by evaluation of MS images. The parameters given in Table 1 are a result of multiple optimization iterations and can directly be applied or further adapted to different tasks. Two methods are given for matrix deposition, varying the speed of solvent being delivered to the surface and therefore putting the emphasis on sensitivity or spatial resolution.

The device is in active use in our laboratory and has replaced all previous manual and automated matrix deposition systems. It has been proven to be robust and reliable in daily operation. Fig. 4 shows an MS image acquired on a FlashQuant system (ABSciex, Toronto) after coating the sample with the sprayer using the standard protocol listed in Table 1. The sample shown is a rat section of an animal dosed at $30 \mathrm{mg} / \mathrm{kg}$ body weight, sacrificed $1 \mathrm{~h}$ after administration. The concentration

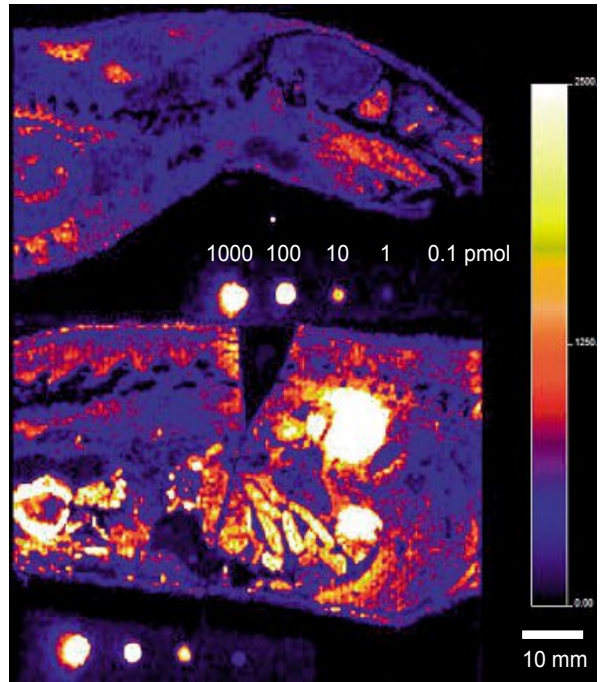

Fig. 4. MS image of a rat section (1 h post dose, $30 \mathrm{mg} / \mathrm{kg}$ ) coated with the protocol listed above. Data acquired on a FlashQuant system in MRM mode.

Table 1. Typical spray parameter for matrix and internal standard. The methods were optimized for best image quality in the MALDI MSI experiment.

\begin{tabular}{|l|l|l|l|}
\hline & Dry spray (resolution) & Wet spray (sensitivity) & Internal standard \\
\hline Solution & $10 \mathrm{mg} / \mathrm{mL} \mathrm{CHCA}$ & $10 \mathrm{mg} / \mathrm{mL}$ CHCA & $50 \mu \mathrm{M}$ standard \\
\hline Solvent & $50: 50 \mathrm{AXN} / \mathrm{H}_{2} \mathrm{O}(\mathrm{v}: \mathrm{v})$ & $50: 50 \mathrm{AXN} / \mathrm{H}_{2} \mathrm{O}(\mathrm{v}: \mathrm{v})$ & $\mathrm{H}_{2} \mathrm{O}$ \\
\hline Spray Height & $60 \mathrm{~mm}$ & $60 \mathrm{~mm}$ & $60 \mathrm{~mm}$ \\
\hline Speed & $180 \mathrm{~mm} / \mathrm{s}$ & $180 \mathrm{~mm} / \mathrm{s}$ & $180 \mathrm{~mm} / \mathrm{s}$ \\
\hline Line distance & $1 \mathrm{~mm}$ & $1 \mathrm{~mm}$ & $1 \mathrm{~mm}$ \\
\hline Density & $2 \mu \mathrm{L} / \mathrm{cm}^{2}$ & $5 \mu \mathrm{L} / \mathrm{cm}^{2}$ & $1 \mu \mathrm{L} / \mathrm{cm}^{2}$ \\
\hline Break & $60 \mathrm{~s}$ & $0 \mathrm{~s}$ & $0 \mathrm{~s}$ \\
\hline Cycles & 5 & 4 & 1 \\
\hline
\end{tabular}


in blood was determined to be $23 \mu \mathrm{M}$ by LCMS. The sample also contains calibration spots on homogenized liver sections (round spots outside of the tissue). One microliter containing $1 \mathrm{nmol}, 100 \mathrm{pmol}, 10$ pmol, 1 pmol and $100 \mathrm{fmol}$ substance were deposited on the liver. The data shown is a raw image without normalization, in a linear color scale. The image shows the advantage of the spray deposition, delivery high signal intensity due to the relatively wet coating procedure, but at the same time minimizing horizontal analyte migration. No leaking is observed to the tape or the plate from the section, and tissues with different concentrations are well defined.

\section{Conclusions}

We built a robust and reliable device for matrix deposition, tailored to MS imaging application (Fig. 5). The device was tested in our laboratory over multiple months and replaced our existing methods. By using open-source software and commonly available hardware we were able to build a cost-efficient device which is versatile to cover many matrix deposition needs. It can be optimized for specific applications, or modified to provide access to new uses. All information is provided as free and open source software and hardware and is therefore open for distribution and modification. The device allows for true pushbutton operation, as no manual interactions are required besides providing the spray solutions and the sample.

In mass spectrometric imaging, sensitivity and spatial resolution are key parameters which strongly depend on the sample

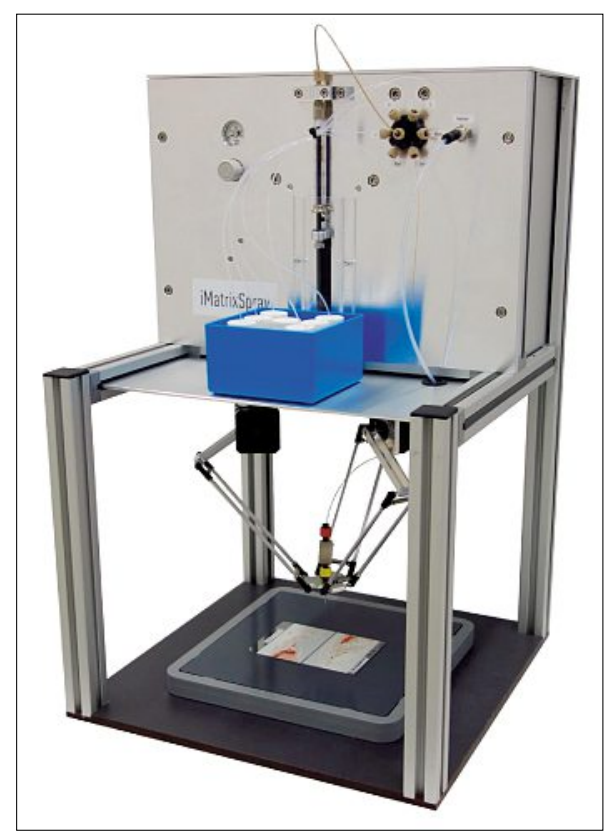

Fig. 5. Prototype of iMatrixSpray device developed under open source software and hardware concept. The device requires power and compressed air and is wirelessly operated over WiFi. The footprint of $40 \times 40 \mathrm{~cm}$ allows for the sprayer to be installed in a fume hood.

preparation technique. The results obtained with the iMatrixSpray demonstrate for the device to perform well in both aspects and therefore for the device to be a new impulse to stimulate MALDI MS Imaging.

\section{Acknowledgements}

The authors are grateful for the support by the COST action BM1104 and thank Lara Schouten for testing the device

Received: February 4, 2014
[1] M. Stoeckli, P. Chaurand, D. E. Hallahan, R. M. Caprioli, Nat. Med. 2001, 7, 493.

[2] L. A. Mcdonnell, R. M. A. Heeren, Mass Spectrom. Rev. 2007, 26, 606.

[3] E. R. Amstalden van Hove, D. F. Smith, R. M. A. Heeren, J. Chromatogr. A 2010, 1217, 3946.

[4] S. A. Schwartz, M. L. Reyzer, R. M. Caprioli, J. Mass Spectrom. 2003, 38, 699.

[5] B. K. Kaletaş, I. M. van der Wiel, J. Stauber, L. J. Lennard, C. Güzel, J. M. Kros, T. M. Luider, R. M. Heeren, Proteomics 2009, 9, 2622.

[6] A. Pirkl, J. Soltwisch, F. Draude, K. Dreisewerd, Anal. Chem. 2012, 84, 5669.

[7] J. Yang, R. Caprioli, Anal. Chem. 2011, 83, 5728.

[8] S. M. Puolitaival, K. E. Burnum, D. S. Cornett, R. M. Caprioli, J. Am. Soc. Mass Spectrom. 2008, $19,882$.

[9] J. A. Hankin, R. M. Barkley, R. C. Murphy, J. Am. Soc. Mass Spectrom. 2007, 18, 1646.

[10] J. Niziol, T. Ruman, Anal. Chem. 2013, 85, 12070.

[11] S. Shimma, Y. Takashima, J. Hashimoto, K. Yonemori, K. Tamura, A. Hamada, J. Mass Spectrom. 2013, 48, 1285.

[12] Y. Chen, Y. Liu, J. Allegood, E. Wang, B. Cachón-González, T. M. Cox, A. H. Merrill Jr, M. C. Sullards, Methods Mol Biol. 2010, 656, 1 .

[13] M. Stoeckli, D. Staab, A. Schweitzer, Int. J. Mass Spectrom. 2007, 260, 195.

[14] R. J. A. Goodwin, A. Nilsson, D. Borg, P. R. R. Langridge-Smith, D. J. Harrison, C. L. Mackay, S. L. Iverson, P. E. Andrén, J. Proteomics 2012, $75,4912$.

[15] A. F. Altelaar, I. Klinkert, K. Jalink, R. P. de Lange, R. A. Adan, R. M. Heeren, S. R. Piersma, Anal. Chem. 2006, 78, 734.

[16] A. Scherl, C. G. Zimmermann-Ivol, J. Di Dio, A. R. Vaezzadeh, P. A. Binz, M. Amez-Droz, R. Cochard, J. C. Sanchez, M. Glückmann, D. F. Hochstrasser, Rapid Commun. Mass Spectrom. 2005, 19, 605 .

[17] D. L. Baluya, T. J. Garrett, R. A. Yost, Anal. Chem. 2007, 79, 6862.

[18] H.-R. Aerni, D. S. Cornett, R. M. Caprioli, Anal. Chem. 2006, 78, 827.

[19] J. M. Pearce, Science 2012, 337, 1303.

[20] iMatrixSpray. http://www.imatrixspray.com/

[21] R. Clavel, Thesis École polytechnique fédérale de Lausanne, 1991. doi:10.5075/epfl-thesis-925

[22] Arduino. http://arduino.cc

[23] RAMP. http://reprap.org/wiki/Arduino_Mega_ Pololu_Shield

[24] Raspberry Pi. http://www.raspberrypi.org

[25] Marlin. https://github.com/ErikZalm/Marlin

[26] OctoPrint. https://github.com/foosel/OctoPrint 